\title{
Dynamics of Entanglement and Nonclassical Correlation for Four-Qubit GHZ State
}

\author{
P. Espoukeh, ${ }^{1}$ R. Rahimi, ${ }^{2}$ S. Salimi,${ }^{3}$ and P. Pedram ${ }^{1}$ \\ ${ }^{1}$ Department of Physics, Science and Research Branch, \\ Islamic Azad University, Tehran, Iran \\ ${ }^{2}$ Institute for Quantum Computing, \\ University of Waterloo, Waterloo, ON, N2L 3G1, Canada \\ ${ }^{3}$ Department of Physics, University of Kurdistan, \\ P.O. Box 66177-15175, Sanandaj, Iran
}

\begin{abstract}
Many-qubit entanglement is crucial for quantum information processing although its exploitation is hindered by the detrimental effects of the environment surrounding the many-qubit system. It is thus of importance to study the dynamics of general multipartite nonclassical correlation, including but not restricted to entanglement, under noise. We did this study for four-qubit GHZ state under most common noises in an experiment and found that nonclassical correlation is more robust than entanglement except when it is imposed to dephasing channel. Quantum discord presents a sudden transition in its dynamics for Pauli-X and Pauli-Y noises as well as Bell-diagonal states interacting with dephasing reservoirs and it decays monotonically for Pauli-Z and isotropic noises.
\end{abstract}

Keywords: Nonclassical correlation; Entanglement; Four-qubit GHZ state; Dynamics under noise 


\section{INTRODUCTION}

It is assumed that entanglement can be useful in quantum computing [1], quantum cryptography [2] and quantum information processing such as superdense coding [3] and quantum teleportation [4]. In practical applications, there are quantum tasks with no entanglement but representing quantum advantages over classical counterparts [5-7]. This indicates that entanglement is not the only feature of quantum correlation and we need to study correlations in a more general concept. This statement is further more correct once it is an experimental implementation of quantum computing or quantum information processing. In any experiment in the field, entanglement is generally difficult to be achieved, but nonclassical correlation is comparably less challenging. Due to this fact, and considering the additional fact that entanglement in larger Hilbert spaces is not well-known yet, understanding the dynamics of nonclassical correlation, generally, and entanglement, specifically, is of importance specially from practical point of view.

Experiments are nowadays elaborated such that they can, at least in some physical systems such as NMR [8] and EPR/ENDOR [9], achieve controls on larger numbers of quits. Therefore, it is getting of practical importance to study the characteristics and behaviour of larger networks of quits and determine the corresponding dynamics of the correlated states under noises that are more common in the physical systems of the study.

Quantum discord is one of the measures of nonclassical correlations [10]. Its definition is based on the difference between two classically equivalent forms of mutual information. It has been shown that quantum discord is very useful to describe correlations involved in quantum systems [11]. Nonclassical correlation and entanglement can have in principal different behaviours. For example, in some temperature ranges, quantum discord of a two-qubit one-dimensional XYZ Heisenberg chain in thermal equilibrium increases with temperature while entanglement decreases with temperature and even it goes to zero [12]. Datta et al. [7] showed that entanglement in DQC1 is negligibly small, however DQC1 includes nonzero discord. Thus, the notion of quantum speed up is related to nonclassical correlation rather than to entanglement [13].

Implementations of quantum computing and quantum information processing are ultimate goals for studies and researches in the field thus there have been continuously extensive efforts in the context of performing experiments relating to quantum technologies. One im- 
mediate consideration that is imposed right after starting plans for experimental practices is how to deal with decoherence of quantum systems. Decoherence is generally critical for any physical system that is determined for experiments, but in the context of quantum protocols this issue becomes one of the most challenging steps. Decoherence of quantum systems are due to unavoidable interactions with the environment and it is led to the degradation of quantum correlations and limitations on using nonclassical correlation and more importantly quantum entanglement in practical applications.

Studies on quantum channels are usually divided into two categories: Markovian [14, 15] and non-Markovian [11, 16, 17]. Investigations of nonclassical correlations under Markovian environments show that nonclassical correlation is more robust than entanglement. Consequently, implementations of quantum algorithms that rely on nonclassical correlation, and not an absolute entanglement, are less fragile [18]. Studies on non-Markovian errors show that since there is no occurrence of sudden death of quantum discord in spite of the entanglement sudden death, the description is more practical in the case of nonclassical correlation [11].

Time evolutions of nonclassical correlation and entanglement in bipartite systems coupled to external environments have been extensively studied [19]. However, this concept is yet not very well resolved for higher dimensions. Quantifications of nonclassical correlation and entanglement for multi-partite systems are not yet generally d known, in addition and consequently determinations of their behavior in presence of environmental noises are far from being clear. In the case of tripartite systems there have been some results [20, 23] but for larger spaces the sources are very few [24 26] and the existing papers only study the entanglement of the states not nonclassical correlations in terms of e.g. quantum discord and their behavior under noise. From the previous studies, we have learned that nonclassical correlation and entanglement are preserved in three-qubit GHZ state under noise, but correlations are eliminated in the case of a W-state [22].

In this paper, we study nonclassical correlation and entanglement of four-qubit Greenberger-Horne-Zeilinger (GHZ) state that is initially prepared in a pure state described as $\left|\mathrm{GHZ}_{4}\right\rangle=\frac{1}{\sqrt{2}}(|0000\rangle+|1111\rangle)$. The time evolutions are studied for the $\left|\mathrm{GHZ}_{4}\right\rangle$ state under experimentally common noises, Pauli-X (bit-flip), Pauli-Y (bit-phase-flip), Pauli-Z (phase-flip) and isotropic (depolarizing) noises. For each type of noise, the time evolution of the state is given by the solution of the master equation in the Lindblad form. The entan- 
glement evolution of the mixed state is described by using the lower bound for multi-qubit concurrence proposed by Li et al. [27]. Nonclassical correlation of the mixed four-qubit GHZ state is described by the global quantum discord that is introduced by Rulli et al. [28] which uses a systematic extension of bipartite quantum discord.

This paper is organized as follows. In the next section, we briefly explain the measures that we use for quantifying entanglement and nonclassical correlation of $\left|\mathrm{GHZ}_{4}\right\rangle$ state. In Sec. III, we obtain time evolutions of entanglement and nonclassical correlation for the initially prepared $\left|\mathrm{GHZ}_{4}\right\rangle$ state but imposed to Pauli and isotropic noises. In Sec. IV] we present the conclusions.

\section{ENTANGLEMENT AND NONCLASSICAL CORRELATION OF $\left|\mathrm{GHZ}_{4}\right\rangle$ STATE}

There is not any general approach in quantifying entanglement for multi-partite states. However, as a practical method, one can make use of entanglement measures for bipartite states, in which characterizing entanglement is simpler, and extend them to all states by convex roof [29]. This approach is generally difficult to be performed analytically. Here, in order to characterize entanglement of $\left|\mathrm{GHZ}_{4}\right\rangle$ state, we apply the lower bound for the multi-

qubit concurrence proposed by Li et al. [27] which is based on the bipartite concurrences of the multi-qubit system corresponding to all possible bipartite cuts of the $N$-qubit system.

For a pure $N$-qubit state, the concurrence is defined as follows

$$
C_{N}(|\psi\rangle)=\sqrt{1-\frac{1}{N} \sum_{i=1}^{N} \operatorname{Tr} \rho_{i}^{2}}
$$

where $\rho_{i}=\operatorname{Tr}(|\psi\rangle\langle\psi|)$ is the reduced density matrix of the $i$-th qubit after tracing out other $N-1$ qubits. The concurrence for mixed $N$-qubit states can be generalized as follows

$$
C_{N}(\rho)=\min \sum_{i} p_{i} C_{N}\left(\left|\psi_{i}\right\rangle\right)
$$

in which the minimum is over the pure-state ensemble that specifies $\rho$, namely, $\rho=$ $\sum_{i} p_{i}\left|\psi_{i}\right\rangle\left\langle\psi_{i}\right|$. Note, that there is no analytical solution to optimize the concurrence of a multi-qubit system except for two-qubit systems and a special case of three-qubit state. 
Recently, Li et al. [27] presented a lower bound to this measure which is simpler to calculate

$$
C_{N}(\rho) \geq \tau_{N}(\rho) \equiv \sqrt{\frac{1}{N} \sum_{n=1}^{N} \sum_{k=1}^{K}\left(C_{k}^{n}\right)^{2}} .
$$

This bound contains $N$ bipartite concurrences $C^{n}$ that correspond to the possible bipartite cuts of the multi-qubit system. In order to calculate each bipartite concurrence $C^{n}$, one needs to evaluate a sum of $K=2^{N-2}\left(2^{N-1}-1\right)$ terms $C_{k}$ that are given by

$$
C_{k}^{n}=\max \left\{0, \lambda_{k}^{1}-\lambda_{k}^{2}-\lambda_{k}^{3}-\lambda_{k}^{4}\right\}
$$

Here, $\lambda_{k}^{i}$ 's $(i=1 . .4)$ are the square roots of the four non-vanishing eigenvalues of the matrix $\rho \tilde{\rho}_{k}^{n}$ in decreasing order and $\tilde{\rho}_{k}^{n}=S_{k}^{n} \rho^{*} S_{k}^{n}$ in which $\rho^{*}$ is the complex conjugate of $\rho$ and $S_{k}^{n}=L_{k}^{n} \otimes L_{0}, k=1, \ldots, K$. Moreover, $L_{0}$ is the generator of the group $S O(2)$ and $L_{k}^{n}$ 's are the generators of the group $S O\left(2^{N-1}\right)$.

Consider the density matrix of a composed system $A B$ being denoted by $\rho . \rho^{A}=\operatorname{Tr}_{B}(\rho)$ and $\rho^{B}=\operatorname{Tr}_{A}(\rho)$ are the reduced density matrices for subsystems $A$ and $B$, respectively. Quantum discord for this system, according to the definition of Ollivier and Zurek, is given by

$$
D(\rho)=I(\rho)-C(\rho)
$$

where $I(\rho)=S\left(\rho^{A}\right)+S\left(\rho^{B}\right)-S(\rho)$ is the quantum mutual information that is a well-known measure of the total correlation and $S(\rho)=-\operatorname{Tr}\left(\rho \log _{2} \rho\right)$ is the von-Neumann entropy. In addition,

$$
C(\rho)=S\left(\rho^{a}\right)-\min _{\left\{\Pi_{k}\right\}} S\left(\rho \mid\left\{\Pi_{k}\right\}\right)
$$

is a measure of classical correlations in which the conditional entropy is defined as $S\left(\rho \mid\left\{\Pi_{k}\right\}\right)=\sum_{k} p_{k} S\left(\rho_{k}\right)$ where $\rho_{k}=\frac{1}{p_{k}}\left(I^{a} \otimes \Pi_{k}^{b}\right) \rho\left(I^{a} \otimes \Pi_{k}^{b}\right)$ is the conditional density operator, $p_{k}=\operatorname{Tr}\left[\left(I^{A} \otimes \Pi_{k}^{B}\right) \rho\right]$, and the minimum is taken over the set of projective measurements $\left\{\Pi_{k}\right\}$. This form of quantum discord is appropriate for bipartite systems and for multi-qubit states we resort to a proper generalization of it.

There are various approaches to generalize quantum discord for multipartite states [3032]. Here, in order to evaluate quantum discord for a four-qubit system, we apply the global quantum discord which is introduced by Rulli et al.. It uses a systematic extension of bipartite quantum discord [28]. The global quantum discord $D\left(\rho_{A_{1} \ldots A_{N}}\right)$ for a multipartite 
state $\rho_{A_{1} \ldots A_{N}}$ is then defined as

$$
D\left(\rho_{A_{1} \ldots A_{N}}\right)=\min _{\left\{\Pi_{k}\right\}}\left[S\left(\rho_{A_{1} \ldots A_{N}} \| \Phi\left(\rho_{A_{1} \ldots A_{N}}\right)\right)-\sum_{j=1}^{N} S\left(\rho_{A_{j}} \| \Phi_{j}\left(\rho_{A j}\right)\right)\right],
$$

where $S\left(\rho_{A_{1} \ldots A_{N}} \| \Phi\left(\rho_{A_{1} \ldots A_{N}}\right)\right)$ is the relative entropy and

$$
\begin{aligned}
\Phi_{j}\left(\rho_{A_{j}}\right) & =\sum_{i} \Pi_{A_{j}}^{i} \rho_{A_{j}} \Pi_{A_{j}}^{i}, \\
\Phi\left(\rho_{A_{1} \ldots A_{N}}\right) & =\sum_{k} \Pi_{k} \rho_{A_{1} \ldots A_{N}} \Pi_{k}, \\
\Pi_{k} & =\Pi_{A_{1}}^{j_{1}} \otimes \ldots \otimes \Pi_{A_{N}}^{j_{N}},
\end{aligned}
$$

and $k$ denotes the index string $\left(j_{1} \ldots j_{N}\right)$.

In order to define local projective measurements, we select a set of von-Neumann measurements as

$$
\Pi_{A_{j}}^{1}=\left(\begin{array}{cc}
\cos ^{2}\left(\frac{\theta_{j}}{2}\right) & e^{i \phi_{j}} \cos \left(\frac{\theta_{j}}{2}\right) \sin \left(\frac{\theta_{j}}{2}\right) \\
e^{-i \phi_{j}} \cos \left(\frac{\theta_{j}}{2}\right) \sin \left(\frac{\theta_{j}}{2}\right) & \sin ^{2}\left(\frac{\theta_{j}}{2}\right)
\end{array}\right)
$$

and

$$
\Pi_{A_{j}}^{2}=\left(\begin{array}{cc}
\sin ^{2}\left(\frac{\theta_{j}}{2}\right) & -e^{-i \phi_{j}} \cos \left(\frac{\theta_{j}}{2}\right) \sin \left(\frac{\theta_{j}}{2}\right) \\
e^{i \phi_{j}} \cos \left(\frac{\theta_{j}}{2}\right) \sin \left(\frac{\theta_{j}}{2}\right) & \cos ^{2}\left(\frac{\theta_{j}}{2}\right)
\end{array}\right)
$$

where $\theta_{j} \in[0, \pi)$ and $\phi_{j} \in[0,2 \pi)$. By varying the angles $\theta_{j}$ and $\phi_{j}$, for $j=1 . .4$, one can find the measurement basis that minimizes Eq. (7).

\section{TIME EVOLUTION OF $\left|\mathrm{GHZ}_{4}\right\rangle$ STATE UNDER NOISE}

Time evolution of a quantum system under noise is given by the master equation in the Lindblad form [33]

$$
\frac{\partial \rho}{\partial t}=-\frac{i}{\hbar}\left[H_{S}, \rho\right]+\sum_{i, \alpha}\left(\mathcal{L}_{i, \alpha} \rho \mathcal{L}_{i, \alpha}^{\dagger}-\frac{1}{2}\left\{\mathcal{L}_{i, \alpha}^{\dagger} \mathcal{L}_{i, \alpha}, \rho\right\}\right),
$$

where $\mathcal{L}_{i, \alpha}=\sqrt{\kappa_{i, \alpha}} \sigma_{\alpha}^{(i)}$ is the Lindblad operator that describes the noise and acts on the $i$ th qubit. $\kappa_{i, \alpha}$ is the decoherence rate and $\sigma_{\alpha}^{(i)}$ are the Pauli spin matrices for the $i$ th qubit with $\alpha=x$ for Pauli-X, $\alpha=y$ for Pauli-Y and $\alpha=z$ for Pauli-Z noise. Also, $H_{S}$ is the 
Hamiltonian of the system. Solutions to the above equation under various noises for fourqubit GHZ state is recently presented in Ref. [34]. The idea is to write the density matrix for infinitesimal time interval $t=\delta t$ by using the Lindblad equation as

$$
\rho(\delta t)=\rho(0)+\left[\sum_{i, \alpha}\left(\mathcal{L}_{i, \alpha} \rho(0) \mathcal{L}_{i, \alpha}^{\dagger}\right)-\frac{1}{2}\left\{\mathcal{L}_{i, \alpha}^{\dagger} \mathcal{L}_{i, \alpha}, \rho(0)\right\}\right] \delta t,
$$

where $\rho(0)=\left|\mathrm{GHZ}_{4}\right\rangle\left\langle\mathrm{GHZ}_{4}\right|$. Then, by using a proper ansatz, the solutions can be obtained for all time [34].

Under different noises, if each qubit interacts locally with the environment then the dynamics of nonclassical correlation and entanglement can be extracted from the above formulation, specifically for an initially prepared four quit GHZ state.

\section{A. Pauli-X noise}

The density matrix of four quit GHZ state under the Pauli-X error is the solution of the Lindblad equation [34], as follows

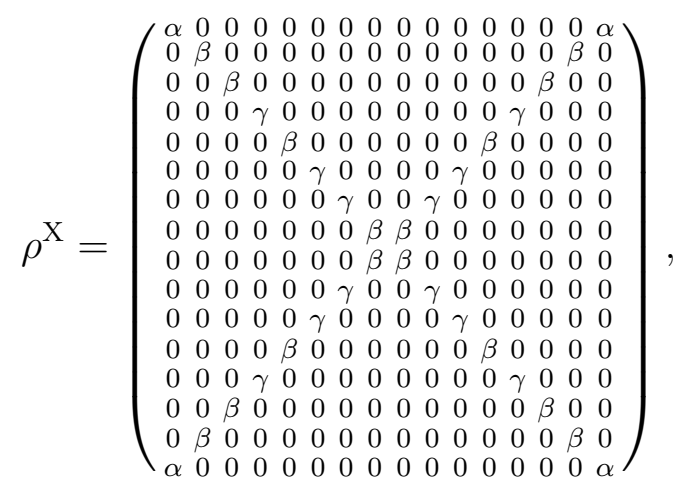

where

$$
\left\{\begin{array}{l}
\alpha=\frac{1}{16}\left(1+6 e^{-4 \kappa t}+e^{-8 \kappa t}\right), \\
\beta=\frac{1}{16}\left(1-e^{-8 \kappa t}\right), \\
\gamma=\frac{1}{16}\left(1-2 e^{-4 \kappa t}+e^{-8 \kappa t}\right),
\end{array}\right.
$$

and $\kappa$ is the decoherence parameter. For this mixed state, the lower bound Eq. (3) to the four-qubit concurrence gives

$$
\tau\left(\rho^{\mathrm{X}}\right)=\max \left\{0, \frac{\sqrt{2}}{4}\left(e^{-8 \kappa t}+6 e^{-4 \kappa t}-3\right)\right\},
$$

which is an exponentially decaying function of time. The plot of this lower bound (Fig. 1) shows that the entanglement of the four-qubit GHZ state vanishes after some finite time for 


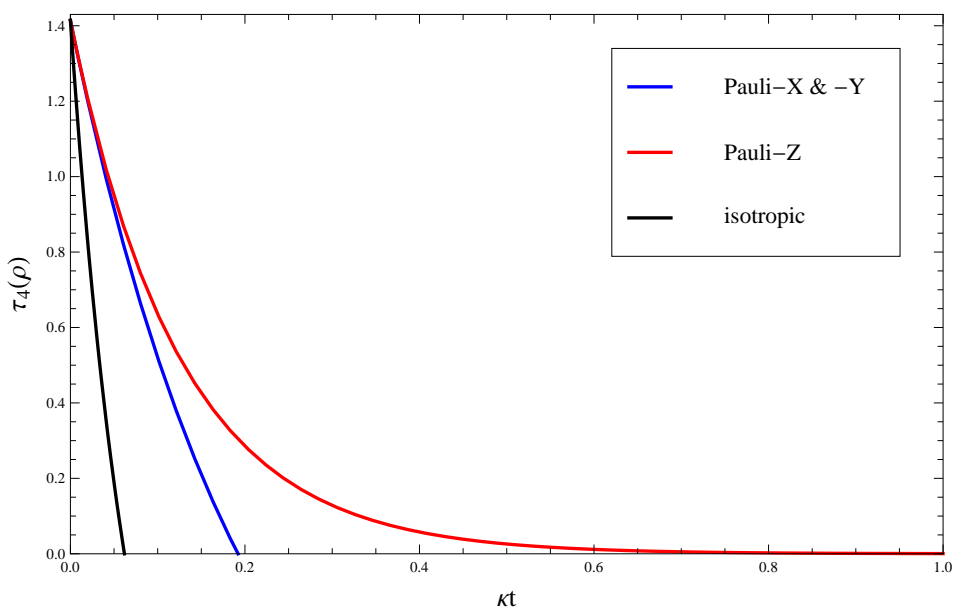

FIG. 1: The lower bound for the four-qubit concurrence for an initial $\left|\mathrm{GHZ}_{4}\right\rangle$ state, transmitted through Pauli-X, Pauli-Y, Pauli-Z and isotropic channels as function of $\kappa t$.

the bit-flip channel. However, using the positive partial transpose criteria [29], we find it out that the density matrix of (15) is separable only for $t \rightarrow \infty$. This indicates that the introduced lower bound does not display proper long-time entanglement for this state.

In order to obtain nonclassical correlation, we need to evaluate Eq. (7) by first finding the reduced density matrix of $i$ th subsystem which is obtained by tracing out other three qubits that reads

$$
\rho_{A_{1}}^{\mathrm{X}}=\rho_{A_{2}}^{\mathrm{X}}=\rho_{A_{3}}^{\mathrm{X}}=\rho_{A_{4}}^{\mathrm{X}}=(\alpha+4 \beta+3 \gamma) \mathbf{1}
$$

Since $\rho_{A_{j}}^{\mathrm{X}}$ is proportional to the unity operator, $\Phi_{j}\left(\rho_{A_{j}}\right)=\sum_{i} \Pi_{A_{j}}^{i} \rho_{A_{j}} \Pi_{A_{j}}^{i}$ is unaffected under any projective measurements, therefore, $\Phi_{j}\left(\rho_{A_{j}}^{\mathrm{X}}\right)=\rho_{A_{j}}^{\mathrm{X}}$ and the second term in Eq. (7) vanishes. To evaluate the first term, we need to find the measurement bases that minimize quantum discord. Calculations show that quantum discord suddenly changes during the dynamics of the system, namely, the measurement bases change from $\sigma_{z}(\theta=0)$ to $\sigma_{x}$ $(\theta=\pi / 2)$, so we find

$$
\begin{aligned}
S\left(\Phi\left(\rho^{\mathrm{X}}\right)\right)= & 4-\frac{\left(1-e^{-8 \kappa t}\right)}{2} \log _{2}\left(1-e^{-8 \kappa t}\right) \\
& -\frac{1}{8}\left[\left(1+6 e^{-4 \kappa t}+e^{-8 \kappa t}\right) \log _{2}\left(1+6 e^{-4 \kappa t}+e^{-8 \kappa t}\right)\right. \\
& \left.+3\left(1-2 e^{-4 \kappa t}+e^{-8 \kappa t}\right) \log _{2}\left(1-2 e^{-4 \kappa t}+e^{-8 \kappa t}\right)\right] .
\end{aligned}
$$

for $0<\kappa t<0.137$ and

$$
S\left(\Phi\left(\rho^{\mathrm{X}}\right)\right)=3,
$$


for $\kappa t>0.137$. Also,

$$
\begin{aligned}
S\left(\rho^{\mathrm{X}}\right)= & 3-\frac{\left(1-e^{-8 \kappa t}\right)}{2} \log _{2}\left(1-e^{-8 \kappa t}\right) \\
& -\frac{1}{8}\left[\left(1+6 e^{-4 \kappa t}+e^{-8 \kappa t}\right) \log _{2}\left(1+6 e^{-4 \kappa t}+e^{-8 \kappa t}\right)\right. \\
& \left.+3\left(1-2 e^{-4 \kappa t}+e^{-8 \kappa t}\right) \log _{2}\left(1-2 e^{-4 \kappa t}+e^{-8 \kappa t}\right)\right] .
\end{aligned}
$$

Finally, we obtain

$$
D\left(\rho^{\mathrm{X}}\right)=1
$$

for $0<\kappa t<0.137$ and

$$
\begin{aligned}
D\left(\rho^{\mathrm{X}}\right)= & \frac{\left(1-e^{-8 \kappa t}\right)}{2} \log _{2}\left(1-e^{-8 \kappa t}\right) \\
& +\frac{1}{8}\left[\left(1+6 e^{-4 \kappa t}+e^{-8 \kappa t}\right) \log _{2}\left(1+6 e^{-4 \kappa t}+e^{-8 \kappa t}\right)\right. \\
& \left.+3\left(1-2 e^{-4 \kappa t}+e^{-8 \kappa t}\right) \log _{2}\left(1-2 e^{-4 \kappa t}+e^{-8 \kappa t}\right)\right],
\end{aligned}
$$

for $\kappa t>0.137$. Therefore, the quantum discord is constant for $0<\kappa t<0.137$, however, it experiences a sudden transition in its dynamics at $\kappa t=0.137$ and monotonically decays for $\kappa t>0.137$. This peculiar behavior of quantum discord is known as the sudden change of the quantum discord which is also observed for two qubit systems [35, 36], linked to the universal freezing of quantum correlations [37].

\section{B. Pauli-Y noise}

Now, we investigate the time evolution of nonclassical correlation and entanglement for $\left|\mathrm{GHZ}_{4}\right\rangle$ state under Pauli-Y error. The density matrix for this system is given by [34]

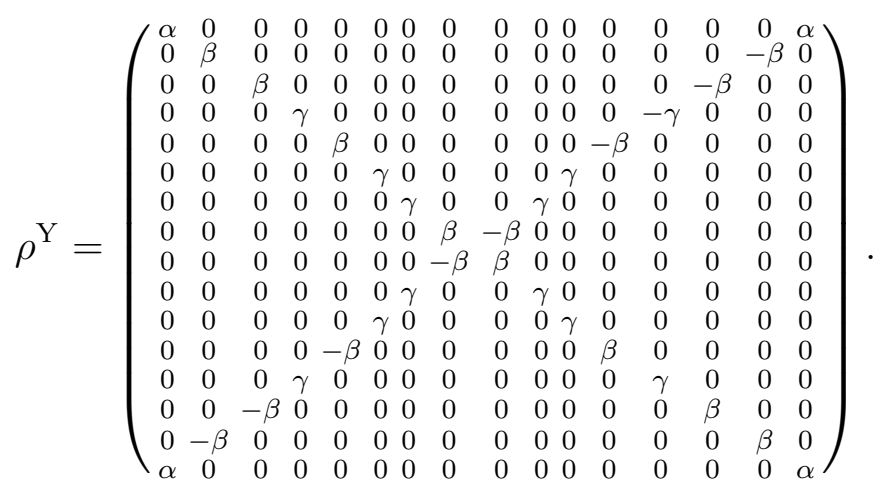

For this case, the lower bound to the concurrence is similar to Eq. (17), i.e., $\tau\left(\rho^{\mathrm{Y}}\right)=$ $\max \left\{0, \frac{\sqrt{2}}{4}\left(e^{-8 \kappa t}+6 e^{-4 \kappa t}-3\right)\right\}$. Also, similar to Pauli-X noise, we observe a sudden change 
in quantum discord at $\kappa t=0.137$. Indeed, for $\kappa t<0.137$ the optimal local measurements are given by $\Pi_{A_{j}}^{i}=|i\rangle\langle i|$ and since $\Phi_{j}\left(\rho_{A_{j}}^{\mathrm{Y}}\right)=\rho_{A_{j}}^{\mathrm{Y}}$ and $S\left(\Phi\left(\rho^{\mathrm{Y}}\right)\right)-S\left(\rho^{\mathrm{Y}}\right)=1$, we find

$$
D\left(\rho^{\mathrm{Y}}\right)=1
$$

But, for $\kappa t>0.137, \sigma_{y}$ is the basis which minimizes quantum discord, so we have $S\left(\Phi\left(\rho^{\mathrm{Y}}\right)\right)=$ 3 and $S\left(\rho^{\mathrm{Y}}\right)=S\left(\rho^{\mathrm{X}}\right)$. Therefore, $D\left(\rho^{\mathrm{Y}}\right)$ coincides with Eq. (23).

\section{Pauli-Z noise}

For the next case, consider Pauli-Z error where its corresponding density matrix takes the following form [34]

$$
\rho^{Z}=\frac{1}{2}\left(|0\rangle^{\otimes 4}\left\langle\left. 0\right|^{\otimes 4}+\mid 1\right\rangle^{\otimes 4}\left\langle\left. 1\right|^{\otimes 4}\right)+\frac{1}{2} e^{-8 \kappa t}\left(|0\rangle^{\otimes 4}\left\langle\left. 1\right|^{\otimes 4}+\mid 1\right\rangle^{\otimes 4}\left\langle\left. 0\right|^{\otimes 4}\right),\right.\right.
$$

that results in the lower bound

$$
\tau\left(\rho^{\mathrm{Z}}\right)=\sqrt{2} e^{-8 \kappa t}
$$

As it is shown in Fig. 1, nonclassical correlation and entanglement of this state decrease more slowly with respect to the previous noise models. For this case, the reduced density matrices become

$$
\rho_{A_{1}}^{\mathrm{Z}}=\rho_{A_{2}}^{\mathrm{Z}}=\rho_{A_{3}}^{\mathrm{Z}}=\rho_{A_{4}}^{\mathrm{Z}}=\frac{\mathbf{1}}{2},
$$

which results in $\Phi_{j}\left(\rho_{A_{j}}^{Z}\right)=\rho_{A_{j}}$, therefore the second term of Eq. (17) vanishes. The optimization procedure of $\theta$ and $\phi$ for each qubit shows that the optimized projective measurements are the bases of $\sigma_{z}$. Then we find $S\left(\Phi\left(\rho^{\mathrm{Z}}\right)\right)=1$ and the von-Neumann entropy becomes

$$
S\left(\rho^{\mathrm{Z}}\right)=1-\frac{1}{2}\left(1-e^{-8 \kappa t}\right) \log _{2}\left(1-e^{-8 \kappa t}\right)+\frac{1}{2}\left(1+e^{-8 \kappa t}\right) \log _{2}\left(1+e^{-8 \kappa t}\right) .
$$

Therefore, we have

$$
D\left(\rho^{\mathrm{Z}}\right)=\frac{1}{2}\left(1-e^{-8 \kappa t}\right) \log _{2}\left(1-e^{-8 \kappa t}\right)+\left(1+e^{-8 \kappa t}\right) \log _{2}\left(1+e^{-8 \kappa t}\right) .
$$

This result shows that the Pauli-Z error causes dissipative behavior on nonclassical correlation for $\left|\mathrm{GHZ}_{4}\right\rangle$ state. Decoherence behavior in terms of $\kappa t$ is depicted in Fig. 2 . 


\section{Isotropic noise}

To this end, consider $\left|\mathrm{GHZ}_{4}\right\rangle$ state under isotropic noise. It should be noted that for this case, the Lindblad equation depends on all three Pauli operators (see Ref. 29). Thus, the density matrix as the solution of the master equation is described by [34]

$$
\rho^{\mathrm{D}}=\left(\begin{array}{cccccccccccccccc}
\tilde{\alpha}_{+} & 0 & 0 & 0 & 0 & 0 & 0 & 0 & 0 & 0 & 0 & 0 & 0 & 0 & 0 & \tilde{\alpha}_{-} \\
0 & \tilde{\beta} & 0 & 0 & 0 & 0 & 0 & 0 & 0 & 0 & 0 & 0 & 0 & 0 & 0 & 0 \\
0 & 0 & \tilde{\beta} & 0 & 0 & 0 & 0 & 0 & 0 & 0 & 0 & 0 & 0 & 0 & 0 & 0 \\
0 & 0 & 0 & \tilde{\gamma} & 0 & 0 & 0 & 0 & 0 & 0 & 0 & 0 & 0 & 0 & 0 & 0 \\
0 & 0 & 0 & 0 & \tilde{\beta} & 0 & 0 & 0 & 0 & 0 & 0 & 0 & 0 & 0 & 0 & 0 \\
0 & 0 & 0 & 0 & 0 & \tilde{\gamma} & 0 & 0 & 0 & 0 & 0 & 0 & 0 & 0 & 0 & 0 \\
0 & 0 & 0 & 0 & 0 & 0 & \tilde{\gamma} & 0 & 0 & 0 & 0 & 0 & 0 & 0 & 0 & 0 \\
0 & 0 & 0 & 0 & 0 & 0 & 0 & \tilde{\beta} & 0 & 0 & 0 & 0 & 0 & 0 & 0 & 0 \\
0 & 0 & 0 & 0 & 0 & 0 & 0 & 0 & \tilde{\beta} & 0 & 0 & 0 & 0 & 0 & 0 & 0 \\
0 & 0 & 0 & 0 & 0 & 0 & 0 & 0 & 0 & \tilde{\gamma} & 0 & 0 & 0 & 0 & 0 & 0 \\
0 & 0 & 0 & 0 & 0 & 0 & 0 & 0 & 0 & 0 & \tilde{\gamma} & 0 & 0 & 0 & 0 & 0 \\
0 & 0 & 0 & 0 & 0 & 0 & 0 & 0 & 0 & 0 & 0 & \tilde{\beta} & 0 & 0 & 0 & 0 \\
0 & 0 & 0 & 0 & 0 & 0 & 0 & 0 & 0 & 0 & 0 & 0 & \tilde{\gamma} & 0 & 0 & 0 \\
0 & 0 & 0 & 0 & 0 & 0 & 0 & 0 & 0 & 0 & 0 & 0 & 0 & \tilde{\beta} & 0 & 0 \\
0 & 0 & 0 & 0 & 0 & 0 & 0 & 0 & 0 & 0 & 0 & 0 & 0 & 0 & \tilde{\beta} & 0 \\
\tilde{\alpha}- & 0 & 0 & 0 & 0 & 0 & 0 & 0 & 0 & 0 & 0 & 0 & 0 & 0 & 0 & \tilde{\alpha}_{+}
\end{array}\right),
$$

where

$$
\left\{\begin{array}{l}
\tilde{\alpha}_{+}=\frac{1}{16}\left(1+6 e^{-8 \kappa t}+e^{-16 \kappa t}\right) \\
\tilde{\beta}=\frac{1}{16}\left(1-e^{-16 \kappa t}\right) \\
\tilde{\gamma}=\frac{1}{16}\left(1-2 e^{-8 \kappa t}+e^{-16 \kappa t}\right) \\
\tilde{\alpha}_{-}=\frac{1}{2} e^{-16 \kappa t}
\end{array}\right.
$$

Now, the lower bound (3) to the concurrence reads

$$
\left.\tau_{(} \rho^{\mathrm{D}}\right)=\max \left\{0, \frac{\sqrt{2}}{8}\left(9 e^{-16 \kappa t}+6 e^{-8 \kappa t}-7\right)\right\},
$$

This lower bound shows strong dissipation of entanglement in presence of isotropic error. It should be noted that for this density matrix, similar to the Pauli-X and Pauli-Y errors, according to the positive partial trace criteria, expression (33) is only valid for $t<t_{0}$.

The reduced density matrices become

$$
\rho_{A_{1}}^{\mathrm{D}}=\rho_{A_{2}}^{\mathrm{D}}=\rho_{A_{3}}^{\mathrm{D}}=\rho_{A_{4}}^{\mathrm{D}}=(\alpha+4 \beta+3 \gamma) \mathbf{1}
$$

So, Eq. (7) reduces to

$$
D\left(\rho_{A_{1} \ldots A_{N}}^{\mathrm{D}}\right)=\min _{\left\{\Pi_{k}\right\}} S\left(\rho_{A_{1} \ldots A_{N}}^{\mathrm{D}} \| \Phi\left(\rho_{A_{1} \ldots A_{N}}^{\mathrm{D}}\right)\right) .
$$


Calculations show that the optimized local projective measurements are $\Pi_{A_{j}}^{i}=|i\rangle\langle i|$. Thus, we have

$$
\begin{aligned}
S\left(\rho^{\mathrm{D}}\right)= & 4-\frac{\left(1-e^{-16 \kappa t}\right)}{2} \log _{2}\left(1-e^{-16 \kappa t}\right) \\
& -\frac{3}{8}\left(1-2 e^{-8 \kappa t}+e^{-16 \kappa t}\right) \log _{2}\left(1-2 e^{-8 \kappa t}+e^{-16 \kappa t}\right) \\
& -\frac{1}{16}\left(1+6 e^{-8 \kappa t}-7 e^{-16 \kappa t}\right) \log _{2}\left(1+6 e^{-8 \kappa t}-7 e^{-16 \kappa t}\right) \\
& +\frac{1}{16}\left(1+6 e^{-8 \kappa t}+9 e^{-16 \kappa t}\right) \log _{2}\left(1+6 e^{-8 \kappa t}+9 e^{-16 \kappa t}\right),
\end{aligned}
$$

and

$$
\begin{aligned}
S\left(\Phi\left(\rho^{\mathrm{D}}\right)\right)= & 4-\frac{\left(1-e^{-16 \kappa t}\right)}{2} \log _{2}\left(1-e^{-16 \kappa t}\right) \\
& -\frac{3}{8}\left(1-2 e^{-8 \kappa t}+e^{-16 \kappa t}\right) \log _{2}\left(1-2 e^{-8 \kappa t}+e^{-16 \kappa t}\right) \\
& -\frac{1}{8}\left(1+6 e^{-8 \kappa t}+e^{-16 \kappa t}\right) \log _{2}\left(1+6 e^{-8 \kappa t}+e^{-16 \kappa t}\right) .
\end{aligned}
$$

By inserting Eqs. (36) and (37) in Eq. (35) then

$$
\begin{aligned}
D\left(\rho^{\mathrm{D}}\right)= & -\frac{1}{8}\left(1+6 e^{-8 \kappa t}+e^{-16 \kappa t}\right) \log _{2}\left(1+6 e^{-8 \kappa t}+e^{-16 \kappa t}\right) \\
& +\frac{1}{16}\left(1+6 e^{-8 \kappa t}-7 e^{-16 \kappa t}\right) \log _{2}\left(1+6 e^{-8 \kappa t}-7 e^{-16 \kappa t}\right) \\
& \left.+\frac{1}{16}\left(1+6 e^{-8 \kappa t}+9 e^{-16 \kappa t}\right) \log _{2}\left(1+6 e^{-8 \kappa t}+9 e^{-16 \kappa t}\right)\right] .
\end{aligned}
$$

The time evolution of quantum discord for this case is plotted in Fig. 2, As the figure shows, the isotropic error results in a more decrease in quantum discord in comparison to Pauli-Z channel.

\section{CONCLUSIONS}

In this paper, we investigated the dynamics of nonclassical correlation and entanglement for the initial $\left|\mathrm{GHZ}_{4}\right\rangle$ state under interactions with several independent Markovian environments. These noises include Pauli-X, Pauli-Y, Pauli-Z and the isotropic errors. In order to describe the time evolution of entanglement, we utilized the lower bound (3) as an approximation for the convex roof of the four-qubit concurrence. The results showed that entanglement is more robust in transmission under the Pauli-Z error compared to Pauli-X, Pauli-Y and isotropic errors. For Pauli-X, Pauli-Y, and isotropic errors the lower bound 


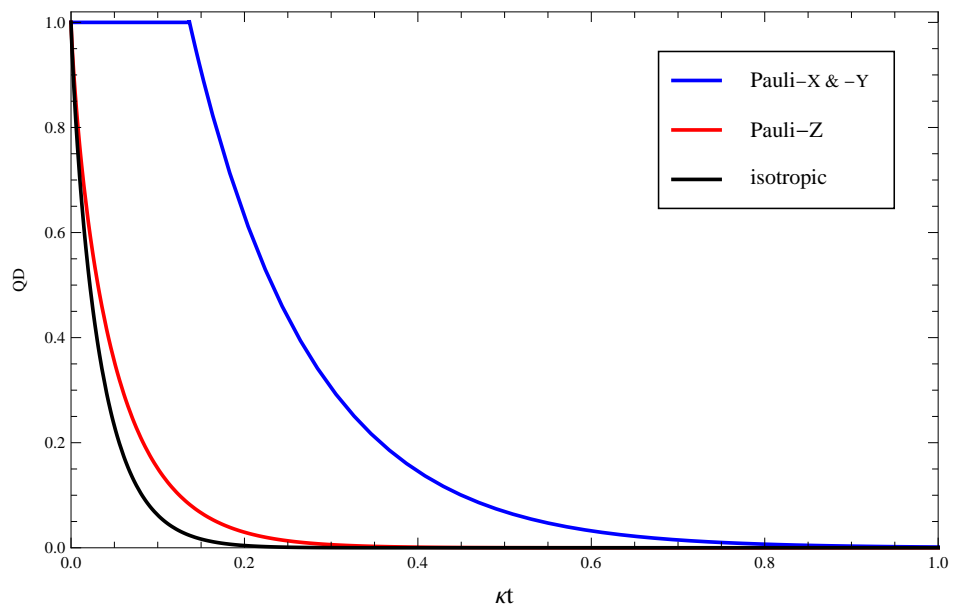

FIG. 2: Quantum discord for the four-qubit system with the initial $\left|\mathrm{GHZ}_{4}\right\rangle$ state transmitted under Pauli-X, Pauli-Y, Pauli-Z, and isotropic errors as a function of $\kappa t$.

vanishes in a finite time (sudden death phenomenon). However, from the positive partial transpose separability criteria [29], we conclude that these states are separable only for $t \rightarrow \infty$. Thus, the lower bound only describes the behavior of the entanglement for $t<t_{0}$ where the lower bound vanishes at $t_{0}$. This is due to the fact that the accuracy of the lower bound over convex roof seems to depend on the rank of the corresponding density matrix [21]. Indeed, the numerical calculation of the convex roof is a quite difficult task which requires an optimization over too many (proportional to $r^{3}$ where $r$ is the rank of the density matrix) free parameters [21].

In order to obtain the time evolution of nonclassical correlation, we used the global quantum discord as a measure to calculate nonclassical correlations in multipartite states. We found that quantum discord is not affected by Pauli-X and Pauli-Y noises for $\kappa t<0.137$ whereas it decreases monotonically for $\kappa t>0.137$. This transition of quantum discord is due to the transition of measurement bases which minimize the quantum discord. Also, quantum discord decays in the presence of Pauli-Z and isotropic noises in terms of the dimensionless scaled time $\kappa t$. In comparison, quantum discord of $\left|\mathrm{GHZ}_{3}\right\rangle$ state is more robust than $\left|\mathrm{GHZ}_{4}\right\rangle$ against decoherence for all noises under study [38]. Moreover, except the case of Pauli-Z error, quantum discord is more robust than entanglement for the initially prepared $\left|\mathrm{GHZ}_{4}\right\rangle$ state. 


\section{Acknowledgments}

RR is supported by CIFAR, Industry Canada and NSERC.

[1] D.P. DiVincenzo, Science 270, 255 (1995).

[2] A.K. Ekert, Phys. Rev. Lett. 67, 661 (1991).

[3] C.H. Bennett and S.J. Wiesner, Phys. Rev. Lett. 69, 2881 (1992).

[4] C.H. Bennett, G. Brassard, C. Crepeau, R. Jozsa, A. Peres, and W.K. Wootters, Phys. Rev. Lett. 70, 1895 (1993).

[5] L.K. Grover, Phys. Rev. Lett. 79, 325 (1997).

[6] E. Knill and R. Laflamme, Phys. Rev. Lett. 81, 5672 (1998).

[7] A. Datta, A. Shaji, and C.M. Caves, Phys. Rev. Lett. 100, 050502 (2008).

[8] C. Negrevergne, T. S. Mahesh, C. A. Ryan, M. Ditty, F. Cyr-Racine, W. Power, N. Boulant, T. Havel, D. G. Cory, and R. Laflamme, Phys. Rev. Lett. 96, 170501 (2006).

[9] R. Rahimi, K. Sato, K. Furukawa, K. Toyota, D. Shiomi, T. Nakamura, M. Kitagawa, and T Takui, Int. J. Quantum Inf. 3, (supp01) 197 (2005).

[10] H. Ollivier and W.H. Zurek, Phys. Rev. Lett. 88, 017901 (2001).

[11] B. Wang, Z.-Y. Xu, Z.-Q. Chen, and M. Feng, Phys. Rev. A 81, 014101 (2010).

[12] T. Werlang and G. Rigolin, Phys. Rev. A 81, 044101 (2010).

[13] K. Modi, H. Cable, M. Williamson, and V. Vedral, Phys. Rev. X 1, 021022 (2011).

[14] A. Streltsov, H. Kampermann, and D. Bruss, Phys. Rev. Lett. 107, 170502 (2011).

[15] J.-S. Xu, X.-Y. Xu, C.-F. Li, C.-J. Zhang, X.-B. Zou, and G.-C. Guo, Nature Commun. 1, 7 (2010).

[16] F.F. Fanchini, T. Werlang, C.A. Brasil, L.G.E. Arruda, and A.O. Caldeira, Phys. Rev. A 81, 052107 (2010).

[17] E.-M. Laine, J. Piilo, and H.-P. Breuer, Phys. Rev. A 81, 062115 (2010).

[18] T. Werlang, S. Souza, F.F. Fanchini, and C.J. Villas Boas, Phys. Rev. A 80, 024103 (2009);

A. Ferraro, L. Aolita, D. Cavalcanti, F.M. Cucchietti, and A. Acin, Phys. Rev. A 81, 052318 (2010).

[19] P. Bordone, F. Buscemi, and C. Benedetti, Fluct. Noise Lett. 11, 1242003 (2012); C. Benedetti, 
F. Buscemi, P. Bordone, and M.G.A. Paris, Int. J. Quantum Inf. 10, (2012) 1241005.

[20] M. Siomau and S. Fritzsche, Phys. Rev. A 82, 062327 (2010).

[21] M. Siomau and S. Fritzsche, Eur. Phys. J. D 60, 397 (2010).

[22] F. Buscemi and P. Bordone, Phys. Rev. A 87, 042310 (2013).

[23] P. Espoukeh and P. Pedram, Quant. Inf. Proc. 14, 303 (2015).

[24] M. Ali, J. Phys. B: At. Mol. Opt. Phys. 47, 055503 (2014).

[25] M. Ali, Phys. Lett. A 378, 2048 (2014).

[26] P. Espoukeh and P. Pedram, Int. J. Quantum Inform. 13, 1550004 (2015).

[27] M. Li, S.-M. Fei, and Z.-X. Wang, J. Phys. A: Math. Theor. 42, 145303 (2009).

[28] C.C. Rulli and M.S. Sarandy, Phys. Rev. A 84, 042109 (2011).

[29] R. Horodecki, P. Horodecki, M. Horodecki, and K. Horodecki, Rev. Mod. Phys. 81, 865 (2009).

[30] I. Chakrabarty, P. Agrawal, and A.K. Pati, Eur. Phys. J. D 65, 605 (2010).

[31] K. Modi, T. Paterek, W. Son, V. Vedral, and M. Williamson, Phys. Rev. Lett. 104, 080501 (2010).

[32] K. Modi and V. Vedral, AIP Conf. Proc. 1384, 69 (2011).

[33] G. Lindblad, Commun. Math. Phys. 48, 119 (1976).

[34] P. Espoukeh and P. Pedram, Quant. Inf. Proc. 13, 1789 (2014).

[35] J. Maziero, L.C. Cleri, R.M. Serra, and V. Vedral, Phys. Rev. A 80, 044102 (2009).

[36] B. Aaronson, R. Lo Franco, G. Compagno, and G. Adesso, New J. Phys. 15, 093022 (2013).

[37] B. Aaronson, R. Lo Franco, and G. Adesso, Phys. Rev. A 88, 012120 (2013).

[38] M. Mahdian, R. Yousefjani, and S. Salimi, Eur. Phys. J. D 66, 133 (2012). 